\title{
Lower dimensional Yang-Mills theory as a laboratory to study the infrared regime
}

\section{Reinhard Alkofer}

Institut für Physik, Karl-Franzens-Universität, Universitätsplatz, 5, A-8010 Graz, Austria

\section{Christian S. Fischer}

Institut für Physik, TU Darmstadt, Schlossgartenstr. 9, D-64289 Darmstadt, Germany

\section{Markus Q. Huber}

Institut für Physik, Karl-Franzens-Universität, Universitätsplatz, 5, A-8010 Graz, Austria

\section{Kai Schwenzer*}

Institut für Physik, Karl-Franzens-Universität, Universitätsplatz, 5, A-8010 Graz, Austria

E-mail: kai.schwenzer@uni-graz.at

\begin{abstract}
Lattice studies of the infrared regime of gauge theories are complicated by the required extensive limits, the performed gauge fixing and the demand for high statistics. Using a general power counting scheme for the infrared limit of Landau gauge SU(N) Yang-Mills theory in arbitrary dimensions we show that the infrared behavior of Greens functions is both qualitatively and quantitatively similar in two, three and four spacetime dimensions. Therefore, lower dimensional lattice simulations can serve as a simplified laboratory to analyze the presently applied approximations and to obtain first results for higher correlation functions.
\end{abstract}

The XXV International Symposium on Lattice Field Theory

July 30 - August 42007

Regensburg, Germany

\footnotetext{
${ }^{*}$ Speaker.
} 
Introduction: Lattice studies of the infrared (IR) regime of Yang-Mills theory, see e.g. [1], are important to understand its genuinely non-pertubative aspects. This is particularly promising since the detailed mechanism for gluon confinement could be connected to directly measurable correlation functions of the colored fields within the Kugo-Ojima [2] and Gribov-Zwanziger [3] scenarios. These rely on a strong IR increase of the ghost dressing function that was predicted by continuum methods [4, 5, 6, 7] and has been clearly confirmed in lattice simulations [1]. Combined efforts via all available methods such as Dyson-Schwinger equations (DSE) [4, 5, 6, 7], renormalization group (RG) techniques [8] and lattice gauge theory studies [1] led during the last years to a coherent picture of the IR regime in Landau gauge. The continuum approaches allow in particular to analyze the IR scaling limit of general Greens functions [9]. These are compatible with a dominance of the gauge fixing part of the action, as argued for in [10]. A simultaneous analysis of DSE and RG methods allowed furthermore to show that this IR fixpoint is unique [11].

Recently, challenging lattice results by several groups on larger lattice sizes [12] suggested that the IR scaling of the gluon propagator might be weaker than predicted by continuum methods and does not show the IR vanishing required within the Gribov-Zwanziger scenario. The finite size corrections have been previously estimated within a Dyson-Schwinger study on a compact manifold [13] and suggest that despite significant corrections the expected IR behavior should be visible with the quite remarkable lattice sizes employed. However, the four dimensional studies still feature significant statistical fluctuations in the IR which are less pronounced in two [14] and three dimensions [15] where deviations from the continuum predictions are not observed.

The discrepancy of the new lattice data with the continuum results can basically have two reasons. First, it is possible that the IR regime requires even more refined numerical methods on the lattice side. As we will argue below, in this case lower dimensional studies should allow to test the involved approximations in a substantially simpler setting. This conclusion stems from a study of the dependence of the IR limit on the spacetime dimension $d$. We establish a manifest power counting scheme for general vertex functions of Landau gauge Yang-Mills theory in arbitrary dimensions and find qualitatively similar results as in the four-dimensional analysis given in [9].

The other reason for the discrepancy could be that the IR behavior predicted by continuum methods - and in particular the value of the IR scaling parameter $\kappa$ - suffers from the presently studied approximations. In an approximation based on the propagator DSEs and using a bare ghost-gluon vertex it takes a value $\kappa \approx 0.595$ in four dimensions. A possible dressing of this vertex could change this value [6], and the continuum prediction would be compatible with the recent lattice results if the IR exponent $\kappa$ is slightly smaller. Therefore, we also discuss the dependence of the dynamical building blocks in the Dyson-Schwinger equations on $\kappa$.

IR exponents for arbitrary $d$ : In the following we will perform a scaling analysis for the IR regime of Yang-Mills theory in arbitrary dimensions. In contrast to the generic IR limit we study here the limit where the coupling $g$ is kept fixed and has no inherent scaling dependence, which is more directly accessible in lattice simulations and has been analyzed in [14, 15]. As in four dimensions [9], the starting point for the IR analysis is the non-renormalization of the ghost-gluon vertex in Landau gauge [16], which implies a finite vertex in the IR [9]. This property depends purely on the transversality of the gluon propagator and is therefore valid in arbitrary dimensions. The IR behavior of the propagators and vertices well below its inherent scale $g^{2 /(4-d)}$ (respectively $\Lambda_{Q C D}$ 


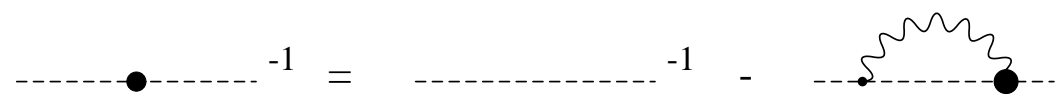

Figure 1: The DSE for the ghost propagator.

in $d=4$ ) is determined via renormalization group arguments by scaling relations. The propagators of the gluons and ghosts

$$
D_{\mu v}\left(p^{2}\right)=\left(\delta_{\mu v}-\frac{p_{\mu} p_{v}}{p^{2}}\right) \frac{Z\left(p^{2}\right)}{p^{2}}, \quad D^{G}\left(p^{2}\right)=-\frac{G\left(p^{2}\right)}{p^{2}}
$$

are given in terms of dressing functions whose IR behavior is described by a power law ansatz

$$
Z\left(p^{2}\right)=c_{0,2} \cdot\left(p^{2}\right)^{\delta_{0,2}}, \quad G\left(p^{2}\right)=c_{2,0} \cdot\left(p^{2}\right)^{\delta_{2,0}},
$$

and similar for the vertices. Here we denote the IR exponent of a vertex with $2 n$ ghost and $m$ gluon legs by $\delta_{2 n, m}$ and the corresponding coefficient by $c_{2 n, m}$. Whereas this coefficient is a constant for the propagators it is generally a function of $2 n+m-1$ momentum ratios.

For the integral on the right hand side of the ghost propagator Dyson-Schwinger equation, cf. fig. 1 , one can use the standard expression $[6,17]$

$$
\int \frac{d^{d} q}{(2 \pi)^{d}}\left(q^{2}\right)^{v_{1}}\left((q-p)^{2}\right)^{v_{2}}=(4 \pi)^{-\frac{d}{2}} \frac{\Gamma\left(\frac{d}{2}+v_{1}\right) \Gamma\left(\frac{d}{2}+v_{2}\right) \Gamma\left(-v_{1}-v_{2}-\frac{d}{2}\right)}{\Gamma\left(-v_{1}\right) \Gamma\left(-v_{2}\right) \Gamma\left(d+v_{1}+v_{2}\right)}\left(p^{2}\right)^{\frac{d}{2}+v_{1}+v_{2}}
$$

which shows that it scales proportional to the external momentum. The left hand side of the ghost DSE, which consists only of the inverse dressed ghost propagator, is proportional to $\left(p^{2}\right)^{-\delta_{2,0}+1}$, where the 1 is the canonical dimension. The scaling dimensions on the right hand side are $d / 2$ from the integral, $\delta_{0,2}-1$ from the gluon propagator, $\delta_{2,0}-1$ from the ghost propagator, $1 / 2$ from the bare ghost-gluon vertex, and $1 / 2$ from the dressed ghost-gluon vertex which features no anomalous scaling. Defining the parameter $\kappa$ as $\kappa:=-\delta_{2,0}$, this condition on the IR exponents yields in four dimensions the well-known result $\delta_{0,2}=2 \kappa$. In $d$ dimensions we have [5, 6]

$$
\delta_{2,0}=-\kappa \quad, \quad \delta_{0,2}=2 \kappa+2-\frac{d}{2} .
$$

In order to transform the infinite hierarchy of Dyson-Schwinger equations into a closed system we perform a skeleton expansion. This yields an infinite tower of graphs involving only primitively divergent vertices which we will analyze in a first step. The first order of the skeleton expansion of the DSE for the three-gluon vertex is depicted in fig. 2.

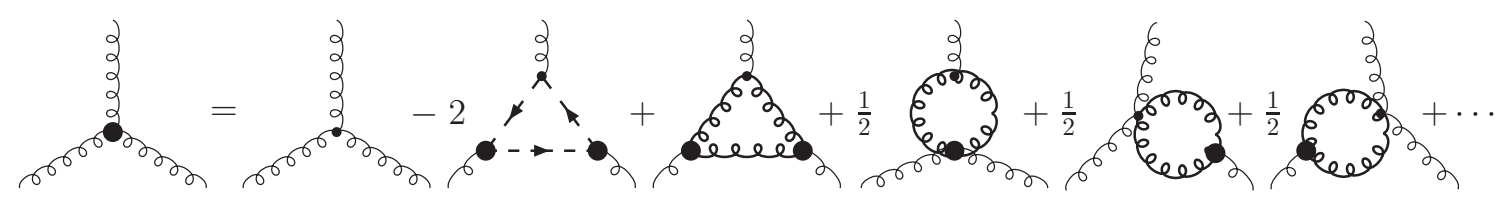

Figure 2: 1-loop part of the skeleton expansion of the DSE for the three-gluon vertex. 

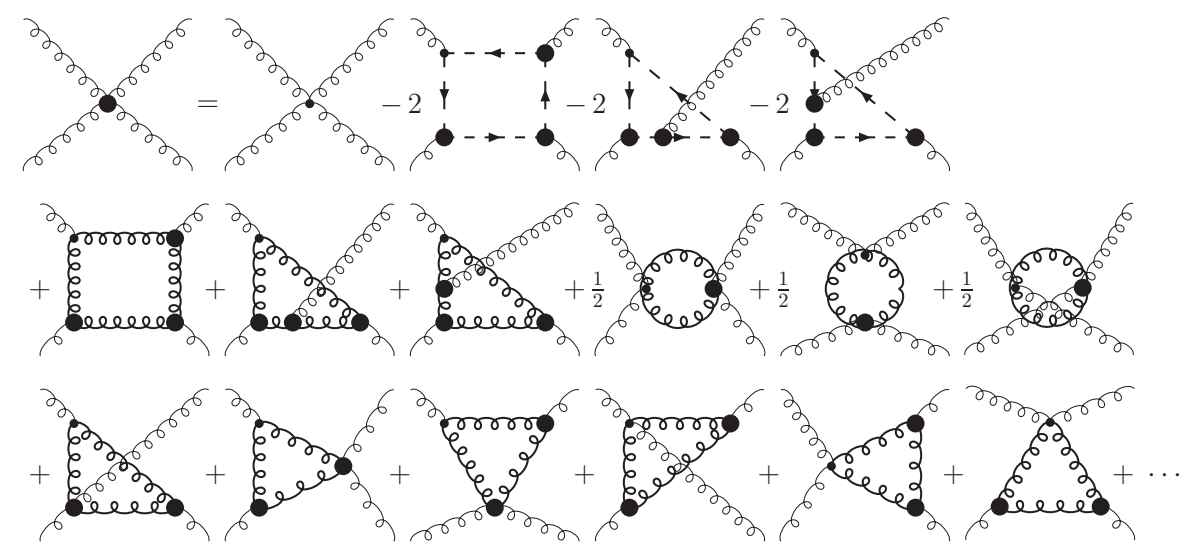

Figure 3: 1-loop part of the skeleton expansion of the DSE for the four-gluon vertex.

We start with the ghost triangle which turns out to be one of the IR leading diagrams. Subtracting the canonical dimension $1 / 2$ we get from the IR counting rules the anomalous IR exponent:

$$
\delta_{0,3}^{g h \Delta}=\frac{d}{2}+3(-\kappa-1)+3 \frac{1}{2}-\frac{1}{2}=-3 \kappa+\frac{d}{2}-2 .
$$

For the four-gluon vertex we can apply the same procedure. In fig. 3 we show the first order of its skeleton expansion. Here, again the ghost rectangle gives one of the IR dominant contributions:

$$
\delta_{0,4}^{g h \square}=\frac{d}{2}+4(-\kappa-1)+4 \frac{1}{2}=-4 \kappa+\frac{d}{2}-2 .
$$

With these results for the scaling of the primitively divergent vertices it is easy to check that the other diagrams in the DSEs fig. 2 and 3 have either the same scaling or are subleading in the IR. Similarly one can analyze the IR exponent of an arbitrary $n$-point function. This is done by counting the IR exponents of the individual building blocks that arise in a general loop correction. The resulting expressions can be simplified via topological identities. It is then straightforward to show that the leading IR exponent of an $n$-point function is given by the simple expression [18]

$$
\delta_{2 n, m}=(n-m) \kappa+(1-n)\left(\frac{d}{2}-2\right) .
$$

This relation verifies a posteriori the assumption discussed above fig. 1: the ghost-gluon scattering kernel is exactly as IR divergent as to make the ghost-gluon vertex finite in the IR, and this is independent of the value of the dimension $d$.

It remains to discuss higher order corrections in the skeleton expansion which can be generated via insertions into given diagrams. The change of the IR exponent due to an exemplary insertion is given by:

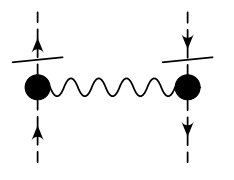

$$
\left(2 \kappa+1-\frac{d}{2}\right)+2(-\kappa-1)+2 \frac{1}{2}+\frac{d}{2}=0,
$$


i.e. the IR-scaling is unchanged. This can also be shown for all other possible extensions [18]. Correspondingly, the skeleton expansion works independent of the number of spacetime dimensions $d[4,5,6,9,11]$. The same holds for the IR limit of the momentum dependent couplings defined through the different vertices: they only feature a scaling via their canonical dimension.

Finally, let us compare the IR behavior of the Yang-Mills Greens functions in different dimensions. We show the IR exponents of the ghost and gluon propagators and the three- and four-gluon vertices in table 1.

\begin{tabular}{l|c|c|c|c} 
Dimension & & 4 & 3 & 2 \\
\hline$\kappa$ & & $0.5953 \ldots \approx 0.6$ & $0.3976 \ldots \approx 0.4$ & 0.2 \\
\hline Ghost & $-\kappa-1$ & -1.6 & -1.4 & -1.2 \\
Gluon & $2 \kappa+1-d / 2$ & 0.2 & 0.3 & 0.4 \\
3-gluon & $-3 \kappa+d / 2-3 / 2$ & -1.3 & -1.2 & -1.1 \\
4-gluon & $-4 \kappa+d / 2-2$ & -2.4 & -2.1 & -1.8
\end{tabular}

Table 1: The dimension dependence of the IR behavior of Yang-Mills Green functions. For each $d$ only the continuum solution for $\kappa$ is displayed that has been qualitatively confirmed by lattice simulations.

Whereas the situation in four dimensions is not fully conclusive, yet, the other solutions have been found in lattice studies in two and three dimensions. These results for the IR exponents of the propagators, the ghost-gluon vertex [19] and the three-gluon vertex in two dimensions [14] agree within errors with the corresponding values given in table 1 . The qualitative behavior does not change in different dimensions and even the quantitative values are very similar. Apart from the listed values of $\kappa$ there is a second branch of solutions of the DSE equation that has not been seen in lattice simulations. We will give some additional arguments below that this branch may be unphysical.

Dependence of the loop integrals on the IR exponent: The DSEs involve loop integrals over bare and dressed vertex functions. As discussed the dressing functions exhibit a power law with appropriate IR exponents. The corresponding coefficients depend on the IR exponent $\kappa$. For the respective coefficients of the propagators, eq. (2), which can be computed from the 2-point integral, eq. (3), this $\kappa$-dependence is shown in fig. 4 , where $\kappa$-independent prefactors have been dropped. The interesting point is, that although the curves in fig. 4 differ considerable, the physical values obtained from the Dyson-Schwinger solution are far away from the poles and thereby the $\kappa$-dependence in their vicinity is rather mild and qualitatively similar in each case.

In general the vertex integrals feature more complicated tensor structures with an increasing number of independent tensor components. However, there are general methods to decompose such tensor integrals to standard scalar integrals [20]. The tensor integrals in the DSE for the ghostgluon and three-gluon vertex reduce to 3-point integrals of the form

$$
I_{3}(p, q) \equiv \int \frac{d^{d} k}{(2 \pi)^{d}} \frac{1}{\left((k+p)^{2}\right)^{v_{1}}} \frac{1}{\left((k-q)^{2}\right)^{v_{2}}} \frac{1}{\left(k^{2}\right)^{v_{3}}}
$$

A general expression for such scalar one-loop 3-point integrals in arbitrary dimensions and with arbitrary powers of the propagators has been obtained in [17, 21]. An analytic expression can be obtained via appropriate series representations for the resulting generalized hypergeometric functions $[22,23]$. Here we will discuss the dependence of the overlap of the tree-level tensor with 

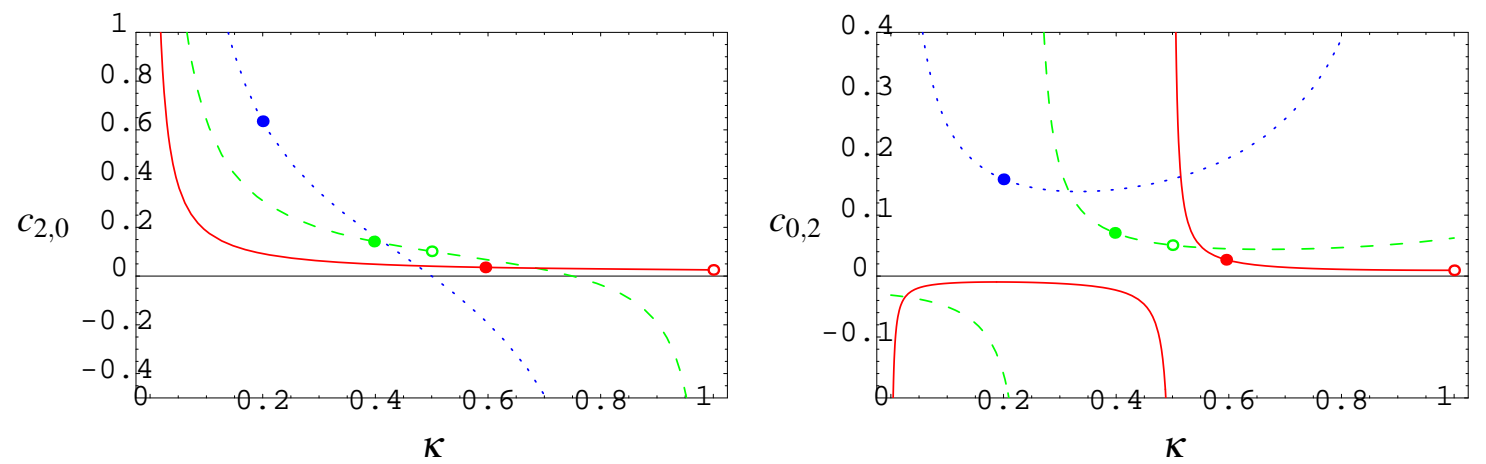

Figure 4: The $\kappa$-dependence of the unrenormalized dressing integrals appearing in the DSEs for the ghost (left) and gluon propagator (right). The dotted, dashed and solid lines show the curves for $d=2,3$ and 4 respectively, see also ref. [6]. The full and open dots represent the solutions of the first and second branch.

the IR-dominant ghost triangle which presents the IR-leading contribution to the Dyson Schwinger equation for the three-gluon vertex in fig. 2. Since this loop involves only ghost-gluon vertices that remain bare to leading order, its IR behavior can be analyzed semi-perturbatively using the scaling form of the dressed ghost propagators, see eq. (2). We consider the special kinematic configuration given by the symmetric point $p^{2}=q^{2}=r^{2}$. At this point the dressing function of the leading ghost loop correction to the three-gluon integral scales according to eq. (5) as $Z_{0,3}=c_{0,3}(\kappa) \cdot\left(p^{2}\right)^{-3 \kappa+d / 2-2}$ where the dependence of the coefficient on the scaling parameter $\kappa$ has been made explicit. The coefficient is shown as a function of $\kappa$ in fig. 5, where the full and open points represent the values for the first and second branch and $\kappa$-independent factors have been dropped again.

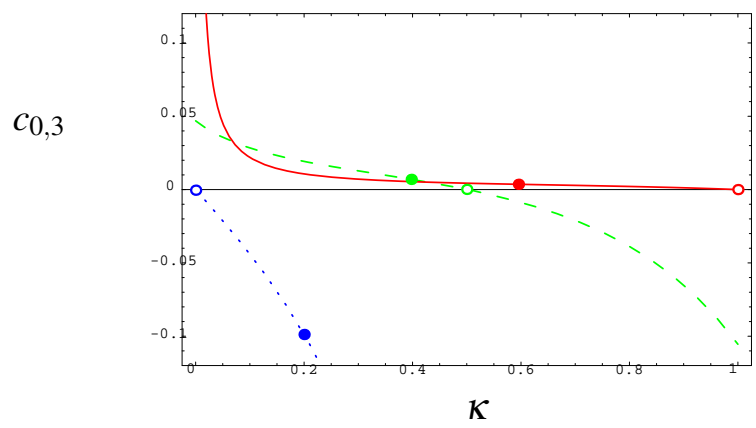

\begin{abstract}
Figure 5: The $\kappa$-dependence of the overlap of the unrenormalized ghost triangle with the tree-level tensor at the symmetric point. The dotted, dashed and solid lines show the curves for $d=2,3$ and 4 respectively. The full and open dots represent the solutions of the first and second branch.
\end{abstract}

The vertex integral has zeros at $\kappa=0$ in two, $\kappa=0.5$ in three and $\kappa=1$ in four dimensions which coincide precisely with the second branch of solutions of the DSEs that is not seen in lattice simulations. Such zeros in the vertex dressing function would lead to an IR-vanishing coupling. Since Yang-Mills theory is apparently a strongly interacting theory, this indicates that these solutions might not be physically relevant. As in the case of the propagators the $\kappa$-dependence in the vicinity of the solutions of the first branch is similar in all dimensions. ynamics would dominate even more.

Conclusions: As in the 4-dimensional case, the IR behavior of Greens functions can be extracted via a skeleton expansion in arbitrary dimensions. The IR limit of Greens functions is surprisingly insensitive on the spacetime dimension. As a consequence Yang-Mills theory in lower dimensions 
should be qualitatively similar to the 4-dimensional theory, and the confinement mechanism might possess identical features. Therefore, corresponding lattice simulations should provide interesting qualitative information for the physical case. Such studies in lower dimensions could in particular provide important lattice results on the IR behavior of vertex functions. This is particularly challenging due to the observation of additional kinematic singularities in current DSE studies [22].

The results on the mild $\kappa$-dependence of the remaining DSE solutions suggest that ghost dominance is a rather robust mechanism and should not depend on the details of the employed truncation scheme. This is further substantiated by the fact that the main non-linearities in the DSE system, which enable the non-trivial fixpoint, arise in the propagator equations whereas there is no non-linear feedback to infrared leading order in the vertex equations.

Acknowledgements: We are grateful to A. Cucchieri, A. Maas, T. Mendez and J. Pawlowski for interesting discussions. This work has been supported in part by the DFG under contract Al279/5-1, by the FWF under contract M979-N16 and by the Helmholtz Association grant VH-NG-332.

\section{References}

[1] A. Sternbeck et al., Phys. Rev. D 72 (2005) 014507 [arXiv:hep-lat/0506007]; A. Cucchieri and T. Mendes, Phys. Rev. D 73 (2006) 071502 [arXiv:hep-lat/0602012]; P. O. Bowman et al., arXiv:hep-lat/0703022; O. Oliveira and P. J. Silva, arXiv:0705.0964 [hep-lat].

[2] T. Kugo and I. Ojima, Prog. Theor. Phys. Suppl. 66 (1979) 1.

[3] V. N. Gribov, Nucl. Phys. B 139 (1978) 1; D. Zwanziger, Nucl. Phys. B 364 (1991) 127.

[4] L. von Smekal, R. Alkofer and A. Hauck, Phys. Rev. Lett. 79 (1997) 3591 [arXiv:hep-ph/9705242];

C. S. Fischer and R. Alkofer, Phys. Lett. B536 (2002) 177 [arXiv:hep-ph/0202202].

[5] D. Zwanziger, Phys. Rev. D 65 (2002) 094039 [arXiv:hep-th/0109224].

[6] C. Lerche and L. von Smekal, Phys. Rev. D 65 (2002) 125006 [arXiv:hep-ph/0202194].

[7] R. Alkofer and L. von Smekal, Phys. Rept. 353 (2001) 281 [arXiv:hep-ph/0007355]; C. S. Fischer, J. Phys. G 32 (2006) R253 [arXiv:hep-ph/0605173].

[8] J. M. Pawlowski et al., Phys. Rev. Lett. 93 (2004) 152002 [arXiv:hep-th/0312324].

[9] R. Alkofer, C.S. Fischer and F.J. Llanes-Estrada, Phys. Lett. B611 (2005) 279[arXiv:hep-th/0412330].

[10] D. Zwanziger, Phys. Rev. D 69 (2004) 016002 [arXiv:hep-ph/0303028].

[11] C. S. Fischer and J. M. Pawlowski, Phys. Rev. D 75 (2007) 025012 [arXiv:hep-th/0609009].

[12] P. O. Bowman et al., arXiv:hep-lat/0703022; I. L. Bogolubsky et al., arXiv:0707.3611 [hep-lat]; A. Cucchieri, and T. Mendes, arXiv:0710.0412 [hep-lat].

[13] C. S. Fischer, B. Gruter and R. Alkofer, Annals Phys. 321 (2006) 1918 [arXiv:hep-ph/0506053]; C. S. Fischer et al., Annals Phys. in print, arXiv:hep-ph/0701050.

[14] A. Maas, Phys. Rev. D 75 (2007) 116004 [arXiv:0704.0722 [hep-lat]].

[15] A. Cucchieri, A. Maas and T. Mendes, Phys. Rev. D 74 (2006) 014503 [arXiv:hep-lat/0605011]; A. Cucchieri, T. Mendes and A. R. Taurines, Phys. Rev. D 67 (2003) 091502 [arXiv:hep-lat/0302022].

[16] J. C. Taylor, Nucl. Phys. B 33 (1971) 436.

[17] C. Anastasiou, E. W. N. Glover and C. Oleari, Nucl. Phys. B 572 (2000) 307 [arXiv:hep-ph/9907494].

[18] M. Huber et al., arXiv:0705.3809 [hep-ph].

[19] A. Cucchieri, T. Mendes and A. Mihara, JHEP 0412 (2004) 012 [arXiv:hep-lat/0408034].

[20] A. I. Davydychev, Phys. Lett. B 263 (1991) 107.

[21] E. E. Boos and A. I. Davydychev, Theor. Math. Phys. 89 (1991) 1052.

[22] R. Alkofer, M. Huber and K. Schwenzer, in preparation.

[23] H. Exton, J. Phys. A: Math. Gen. 28 (1995) 631. 